\title{
Review of Comorbidities of Hidradenitis Suppurativa: Implications for Daily Clinical Practice
}

Thrasyvoulos Tzellos · Christos C. Zouboulis

Received: November 26, 2019 / Published online: January 18, 2020

(C) The Author(s) 2020

\section{ABSTRACT}

Hidradenitis suppurativa (HS) is a chronic, recurrent skin inflammatory disease associated with a variety of comorbidities, like reduced quality of life, metabolic syndrome, sexual dysfunction, working disability, axial spondyloarthritis, inflammatory bowel disease, depression, and anxiety. Like psoriasis, HS

Enhanced Digital Features To view enhanced digital features for this article go to https://doi.org/10.6084/ m9.figshare.11527836.

T. Tzellos $(\bowtie)$

Department of Dermatology, Nordland Hospital

Trust, Bodø, Norway

e-mail: thrasyvoulos.tzellos@unn.no

T. Tzellos

Institute of Clinical Medicine, University of Tromsø,

Tromsø, Norway

C. C. Zouboulis

Departments of Dermatology, Venereology,

Allergology and Immunology, Dessau Medical

Center, Brandenburg Medical School, Theodor

Fontane, Dessau, Germany

C. C. Zouboulis

European Hidradenitis Suppurativa Foundation

e.V., Dessau, Germany patients have been found to have higher risk of cardiovascular death and suicide risk. Clinicians should be informed about these comorbidities so that appropriate screening is implemented. All this evidence suggests that for such a chronic, multi-comorbid disease, the use of validated outcomes to assess severity and effect of treatment, along with the use of clinically important patient reported outcomes, is essential. The potential of available treatments to negatively and positively affect these comorbidities should also be taken into account when designing treatment strategies. This review provides an outline of important HS comorbidities with emphasis on possible implications for daily clinical practice.

Keywords: Clinical significance; Comorbidities; Depression; Hidradenitis suppurativa; Inflammatory bowel disease; Metabolic syndrome; Quality of life; Working disability 


\section{Key Summary Points}

Hidradenitis suppurativa is a chronic, recurrent skin inflammatory disease associated with significant psychological burden, like reduced quality of life, depression, anxiety, and sexual dysfunction.

Important comorbidities include working disability, metabolic syndrome, inflammatory bowel disease and axial spondyloarthritis.

Hidradenitis suppurativa patients are at higher risk of cardiovascular death and suicide risk.

Appropriate screening in daily clinical practice should be implemented for early identification and evidence based treatment.

The use of validated outcomes for assessment of disease severity and treatment effect, along with the use of important patient reported outcomes, is essential.

\section{INTRODUCTION}

Hidradenitis suppurativa (HS), or acne inversa, is a chronic inflammatory skin disease characterized by recurrent inflammation and deepseated lesions in apocrine gland-bearing intertriginous areas of the body that can be potentially scarring and debilitating [1-3]. Prevalence of HS varies widely from 0.03 to $8 \%$, depending on the setting and methodology used [4]. Patients often experience long delays before an HS diagnosis is established [5]. HS typically develops between puberty and the age of 40 years, thus impacts individuals at working age [2]. It is known to have substantial impact on several general health-related and skinspecific quality of life domains [6, 7], is associated with a significant comorbidity burden [3] and overall mortality, and is becoming more widely recognized as an inflammatory disease with a number of systemic implications, like psoriasis.

In a recent global patient survey it became evident that patients reported a high frequency of comorbidities and that they were dissatisfied with medical or procedural treatments in $45.9 \%$ and $34.5 \%$, respectively [8]. For such a chronic, debilitating inflammatory skin disease it is important that clinicians are well informed on possible comorbidities that can have a significant impact. The aim of the review is to present an up-to-date overview of HS comorbidities, discuss their clinical significance and how they can be addressed meaningfully, and shape daily treatment decisions (Table 1). This article is based on previously conducted studies and does not contain any studies with human participants or animals performed by any of the authors.

\section{INCREASED CARDIOVASCULAR (CV) DISEASE RISK}

A meta-analysis of observational studies including $6174 \mathrm{HS}$ patients and 24,993 controls detected significant association of HS with obesity, central obesity, active smoking, history of smoking, hypertriglyceridemia, low HDL, diabetes, and metabolic syndrome [9]. These associations were significant both in population HS patients and hospital HS groups, with hospital HS groups having uniformly higher odds ratios than the population HS groups. CV risk factors appear at a significantly higher rate in HS patients compared to controls. Increased CV risk is manifest even in patients identified in population samples. Obesity is also a wellestablished independent risk factor for HS with improvements in disease severity demonstrated after weight loss, like lower HS prevalence and severity, better long-term prognosis, spontaneous resolution of $\mathrm{HS}$, flare reduction and reduced recurrence after $\mathrm{CO}_{2}$ laser surgery [10-13].

In a cross-sectional study with 68 HS patients and 136 age- and sex-matched healthy control subjects, HS was significantly related to presence of carotid plaques and increased frequency 
Table 1. Important HS comorbidities and their implications for daily clinical practice

\section{Comorbidities}

Association with cardiovascular disease risk factors: obesity, smoking, hypertriglyceridemia, low HDL, diabetes, metabolic syndrome [9]

Increased frequency of subclinical atherosclerosis [14]

Increased risk of adverse cardiovascular outcomes [16]

Association with slower income growth, higher risk of leaving the workforce, more total work loss days, higher annual total indirect costs and lower annual income [17-20]

Axial spondyloarthritis

Association with increased risk of spondyloarthritis [23]

In axial spondyloarthritis patients disease activity score is independently associated with presence of HS [24]

Significant associations of HS with CD and ulcerative colitis [25]

HS patients are at risk for CD [26]

Reduced quality of life, social isolation $[28,29]$

Sexual dysfunction [31]

Depression, anxiety, schizophrenia [29, 34, 35]

Increased antidepressant drug use [36]

Increased suicide risk [36]

Syndromic HS: [36]

PAPA: pyoderma gangrenosum, acne, pyogenic arthritis

PASH: pyoderma gangrenosum, acne, and HS

PAPASH: pyoderma gangrenosum, acne, pyogenic arthritis, and $\mathrm{HS}$

Other

Long-term opioid use [38]

Psoriasis [39], pyoderma gangrenosum [40]

Alopecia areata [40], vitiligo [41]

rheumatoid arthritis [40], non melanoma skin cancer [42]

\section{Clinical significance}

Need for screening for modifiable cardiovascular risks

Avoid treatments with potential negative impact on cardiovascular disease risk factors, e.g., acitretin

Implement weight loss strategies

HS patients have unmet disease management needs

Major surgery in selected cases may improve activity and overall work impairment [21]

Adalimumab significantly improved total work impairment compared to placebo [22]

HS patients with osteoarticular symptoms (low back pain, dactylitis) should be monitored for spondyloarthritis

Axial spondyloarthritis patients with HS may have higher disease activity

Consultation with gastroenterologists if HS patients have recurrent abdominal pain, chronic diarrhea, bloody stool, or body weight loss

Development of strategies to recognize and treat those psychiatric comorbidities in patients with HS is warranted

Lack of response to standard treatment modalities

Early identification is critical in order to optimize management

Older patients, smokers, and patients with depression may benefit from periodic screening for long-term opioid use

$H S$ hidradenitis suppurativa, $C D$ Crohn's disease 
of subclinical atherosclerosis [14]. In a crosssectional Danish general population study, mean resting heart rate in patients with severe HS was significantly higher compared with controls [15]. A population-based cohort study using individual-level linkage of nationwide administrative registers indicated that HS was associated with a significantly increased risk of adverse CV outcomes and all-cause mortality independent of measured confounders [16]. Notably, the risk of CV death was 58\% higher in patients with HS than in patients with severe psoriasis.

These results emphasize the need for screening of HS patients for modifiable CV risk factors and consideration of them when deciding treatment options. For example, acitretin would be better avoided as a treatment for an HS patient with dislipidemia, since an abnormal lipid profile is a well-known potential side effect. Weight gain is a usual side effect of hormonal treatment (ethinyloestradiol and cyproterone acetate), especially with long-term use. Pre-treatment screening should be performed where necessary. Furthermore, weight reduction strategies appear to be beneficial and should be implemented.

\section{WORKING DISABILITY}

HS has been demonstrated to have a significant impact on patients' work productivity and negatively affect opportunities for career advancement. In a Polish survey of employed patients with HS, 58\% reported that the disease caused a work absence with a mean 33.6 days off work annually, $10 \%$ reported that they lost their job because of HS and 23\% believed that HS interfered with promotion [17]. In addition, patients with HS experience a substantial economic burden, with the total direct healthcare costs of patients with HS estimated to be $\$ 6783$ higher compared to those of controls over a 3 -year period [18]. Higher total work productivity impairment scores compared to psoriasis were reported for HS [19]. In a US claims database analysis, newly diagnosed HS patients had significantly slower income growth and higher risk of leaving the workforce compared with controls. General HS patients had more total work loss days, higher annual total indirect costs, and lower annual income compared with controls [20].

This evidence clearly indicates that HS patients have unmet disease management needs with respect to working disability. A single center prospective survey study conducted among 40 patients undergoing major surgery suggested that, after major surgery activity, overall work impairment showed considerable improvement [21]. Individual patient data from two phase 3, randomized, double-blinded studies, PIONEER I and II, and their open-label extension (OLE) trial were used to compare patients with moderate to severe HS treated with adalimumab or placebo [22]. Patients with HS receiving adalimumab experienced significantly lower cumulative indirect costs and significant improvement in total work impairment compared to placebo [22].

\section{AXIAL SPONDYLOARTHRITIS}

A single center cross-sectional study showed that HS was significantly associated with an increased risk of spondyloarthritis, independently of age and sex [23]. A cross-sectional study using a cohort of axial spondyloarthritis patients identified that HS was more prevalent that in the general population and that a higher score on axial spondyloarthritis disease activity score was independently associated with HS [24].

Patients with HS presenting osteoarticular symptoms, especially low back pain or dactylitis, should be monitored for spondyloarthritis. Furthermore, axial spondyloarthritis patients with HS may have higher disease activity.

\section{INFLAMMATORY BOWEL DISEASE}

A recently published meta-analysis of observational studies clearly showed significant associations of HS with Crohn's disease (CD) and ulcerative colitis (UC) [25]. Cross-sectional analysis of data from 51,340 patients with HS, identified using electronic health records data, 
suggested that HS patients are at risk for CD and that prevalence of $\mathrm{CD}$ was greatest among patients with HS who were white, aged 45-64 years, non-obese and tobacco smokers [26]. High HS prevalence has been detected in patients with perianal fistula [27].

In patients with chronic perianal and perineal HS, and in particular in the presence of fistulas, the possibility of CD should be considered. Consultation with gastroenterologists should be sought when patients with HS present with recurrent abdominal pain, chronic diarrhea, bloody stools, and body weight loss. Early detection of the coexistence can lead to appropriate anti-inflammatory treatment to reduce symptoms and disease progression.

\section{PSYCHOLOGICAL COMORBIDITY}

Psychological comorbidities are well established and proven in HS patients. It was suggested that more than $50 \%$ of HS patients experience a very or extremely large effect of HS on quality of life, as measured by the Dermatology Life Quality Index (DLQI) [28]. Using EuroQoL-5D and DLQI scores it was found that HS is in the top five skin diseases with the most negatively affected quality of life and that predictive factors were young age at onset and more lesions per month [29]. DLQI was significantly worse in females compared to males. Quality of life in HS patients was found to be more negatively impacted than in psoriasis, acne, stroke, or heart transplant candidates [28].

It was found that HS has a great emotional impact on patients and promotes isolation due to fear of stigmatization [30]. Body image dissatisfaction may result in feelings of shame, embarrassment, and anxiety and cause lack of self-confidence, depression, and social isolation. Shame and irritation are frequent and relate to smell, scars, itching, and pain [30, 31].

Significant impairment of sexual health has also been clearly indicated in HS patients compared with age-, sex-, and BMI-matched controls, with women affected more than men [28]. Sexual health was impacted in HS due to inconvenience caused by skin inflammation, physical appearance, diminished sexual desire, fear of passing HS on to children and diminished desire of partner [31]. Risk factors for sexual dysfunction for women were education status, disease activity, pain and odor, and lack of a stable relationship; and for men the risk factors were increasing age, active genital lesion and number of active areas [32, 33]. These results reinforce the need to take sexual health into account when assessing disease severity, response to treatment, and patient goals of care.

A recent meta-analysis of the literature suggested that depression and anxiety were very common comorbid conditions in HS patients. Depression was found to be correlated with disease activity and an association between inflammation (CRP levels) and depression has also been identified [29, 34]. Recent evidence from a nationwide population-based study suggested that HS was associated with schizophrenia, after adjusting for demographic factors and smoking status [35].

Taking into account all the abovementioned evidence, it is no surprise that data from a crosssectional and cohort study, using Danish national registries, found that patients with HS compared with the general population had an increased risk of antidepressant drug use, completed suicides and suicidal behavior, and that this increased risk of completed suicide remained, after adjustment for confounding factors [36].

\section{SYNDROMIC HS}

Syndromic HS exists as well. Autoinflammatory syndromes associated with hidradenitis suppurativa (HS) and/or acne are rare but potentially debilitating disorders. Several clinically different syndromes exist including pyoderma gangrenosum, acne, and pyogenic arthritis (PAPA), pyoderma gangrenosum, acne, and hidradenitis suppurativa (PASH), pyoderma gangrenosum, acne, pyogenic arthritis, and hidradenitis suppurativa (PAPASH), psoriatic arthritis, pyoderma gangrenosum, acne, and hidradenitis suppurativa (PsAPASH) and pyoderma gangrenosum, acne and ulcerative colitis (PAC) [37]. 
Important features in these syndromes are painful arthritis, skin lesions (HS, acne and pyoderma gangrenosum) and recurrent episodes of fever. Treatment can be challenging due to lack of response to standard treatment modalities. Early identification is critical in order to optimize management.

\section{OTHER COMORBIDITIES}

A retrospective cohort study showed that HS patients were at higher risk for long-term opioid use [38]. Older patients, smokers, and patients with depression were at higher risk for opioid abuse. Evidence suggests that this subgroup of patients may benefit from periodic screening for long-term opioid use.

Association with psoriasis [39], alopecia areata [40], vitiligo [41], pyoderma gangrenosum, rheumatoid arthritis, and non-melanoma skin cancer [42] have also been suggested as comorbid diseases.

\section{DISCUSSION}

The abovementioned evidence clearly indicates that HS is a multifaceted disease with many different comorbidities that, if not identified early and treated properly, can result in a significant burden for HS patients and society. In order to cope with such a disease it is of high importance to use validated outcomes to assess the severity of the disease and treatment effect.

IHS4 is a validated tool to dynamically assess HS severity and can be used both in real life and in a clinical trials setting. The IHS4 score is calculated by the number of nodules (multiplied by 1) plus the number of abscesses (multiplied by 2) plus the number of draining tunnels (multiplied by 4). A total score of 3 or less signifies mild, 4-10 signifies moderate, and 11 or higher signifies severe disease. This correlates well with the Hurley classification, Expert Opinion, Physician's Global Assessment, Modified Sartorius score, and DLQI [43].

HiSCR (hidradenitis suppurativa clinical response) is defined as $\geq 50 \%$ reduction in inflammatory nodule and abscess count and no increase in number of abscesses or draining fistulas compared with baseline [44]. The use of HiSCR is supported by good-quality validation studies, and is recommended to be used as a dichotomous outcome measure to assess the effects of anti-inflammatory treatment [44]. A post hoc analysis of integrated data from two phase 3 clinical trials (PIONEER I and II) revealed that more HiSCR responders than nonresponders experienced clinically meaningful improvement in the Dermatology Life Quality Index, Pain Numeric Rating Scale, HS quality of life, work-related performance, and non-workrelated performance. Clinically meaningful outcomes in HS are more likely to be attained in patients achieving HiSCR-level improvement.

Patient-reported outcome measures [e.g., DLQI, Numerical Rating Scale (NRS)] should be included in the overall assessment of the HS patient as they may offer important insight on functioning, quality of life, and symptoms (e.g., pain and itching), especially when the minimum clinically important differences for such outcomes is used, like at least a four-unit decrease in DLQI score and $\geq 30 \%$ reduction in NRS pain score.

\section{ACKNOWLEDGEMENTS}

Funding. No funding or sponsorship was received for this study or publication of this article. The Departments of Dermatology, Venereology, Allergology and Immunology, Dessau Medical Center, Brandenburg Medical School Theodor Fontane, Dessau, Germany is a health care unit of the European Reference Network for Rare and Complex Skin Diseases (ERN Skin)

Authorship. All named authors meet the International Committee of Medical Journal Editors (ICMJE) criteria for authorship for this article, take responsibility for the integrity of the work as a whole, and have given their approval for this version to be published. 
Disclosures. Thrasyvoulos Tzellos has acted as advisory board member for AbbVie and UCB. Christos Zouboulis received honoraria from AbbVie, Bayer Healthcare, Biogen and PPM for participation as an advisor and speaker; from Allergan, Almirall, Celgene, GSK, Inflarx, Novartis and UCB for participation as an advisor; and from Jenapharm and Pierre Fabre for participation as a speaker.

Compliance with Ethics Guidelines. This article is based on previously conducted studies and does not contain any studies with human participants or animals performed by any of the authors.

Open Access. This article is licensed under a Creative Commons Attribution-NonCommercial 4.0 International License, which permits any non-commercial use, sharing, adaptation, distribution and reproduction in any medium or format, as long as you give appropriate credit to the original author(s) and the source, provide a link to the Creative Commons licence, and indicate if changes were made. The images or other third party material in this article are included in the article's Creative Commons licence, unless indicated otherwise in a credit line to the material. If material is not included in the article's Creative Commons licence and your intended use is not permitted by statutory regulation or exceeds the permitted use, you will need to obtain permission directly from the copyright holder. To view a copy of this licence, visit http://creativecommons.org/licenses/by$\mathrm{nc} / 4.0 /$.

\section{REFERENCES}

1. Jemec GB. Clinical practice. Hidradenitis suppurativa. N Engl J Med. 2012;366:158-64.

2. Zouboulis CC, Del Marmol V, Mrowietz U, Prens EP, Tzellos T, Jemec GB. Hidradenitis suppurativa/acne inversa: criteria for diagnosis, severity assessment, classification and disease evaluation. Dermatology. 2015;231:184-90.
3. Fimmel S, Zouboulis CC. Comorbidities of hidradenitis suppurativa (acne inversa). Dermatoendocrinol. 2010;2:9-16.

4. Jemec GB, Kimball AB. Hidradenitis suppurativa: epidemiology and scope of the problem. J Am Acad Dermatol. 2015;73:S4-7.

5. Saunte DM, Boer J, Stratigos A, et al. Diagnostic delay in hidradenitis suppurativa is a global problem. Br J Dermatol. 2015;173:1546-9.

6. Gooderham M, Papp K. The psychosocial impact of hidradenitis suppurativa. J Am Acad Dermatol. 2015;73:19-22.

7. Riis PT, Vinding GR, Ring HC, Jemec GB. Disutility in patients with hidradenitis suppurativa: a crosssectional study using EuroQoL-5D. Acta Derm Venereol. 2016;96:222-6.

8. Garg A, Neuren E, Cha D, et al. Evaluating patients' unmet needs in hidradenitis suppurativa: results from the Global VOICE project. J Am Acad Dermatol. 2019. https://doi.org/10.1016/j.jaad.2019. 06.1301.

9. Tzellos T, Zouboulis CC, Gulliver W, Cohen AD, Wolkenstein P, Jemec GB. Cardiovascular disease risk factors in patients with hidradenitis suppurativa: a systematic review and meta-analysis of observational studies. $\mathrm{Br} \mathrm{J}$ Dermatol. 2015;173: 1142-55.

10. Canoui-Poitrine F, Revuz JE, Wolkenstein P, et al. Clinical characteristics of a series of 302 French patients with hidradenitis suppurativa, with an analysis of factors associated with disease severity. J Am Acad Dermatol. 2009;61:51-7.

11. Kromann CB, Ibler KS, Kristiansen VB, Jemec GB. The influence of body weight on the prevalence and severity of hidradenitis suppurativa. Acta Derm Venereol. 2014;94:553-7.

12. Thomas CL, Gordon KD, Mortimer PS. Rapid resolution of hidradenitis suppurativa after bariatric surgical intervention. Clin Exp Dermatol. 2014;39: $315-7$.

13. Boer J. Resolution of hidradenitis suppurativa after weight loss by dietary measures, especially on frictional locations. J Eur Acad Dermatol Venereol. 2016;30:895-6.

14. González-López MA, Hernández JL, Lacalle M, et al. Increased prevalence of subclinical atherosclerosis in patients with hidradenitis suppurativa (HS). J Am Acad Dermatol. 2016;75:329-35.

15. Juhl CR, Miller IM, Jemec GB, Kanters JK, Ellervik C. Hidradenitis suppurativa and electrocardiographic 
changes: a cross-sectional population study. $\mathrm{Br} \mathrm{J}$ Dermatol. 2018;178:222-8.

16. Egeberg A, Gislason GH, Hansen PR. Risk of major adverse cardiovascular events and all-cause mortality in patients with hidradenitis suppurativa. JAMA Dermatol. 2016;152:429-34.

17. Matusiak L, Bieniek A, Szepietowski JC. Hidradenitis suppurativa markedly decreases quality of life and professional activity. J Am Acad Dermatol. 2010;62:706-8.

18. Kirby JS, Miller JJ, Adams DR, Leslie D. Health care utilization patterns and costs for patients with hidradenitis suppurativa. JAMA Dermatol. 2014;150:937-44.

19. Hamzavi IH, Sundaram M, Nicholson C, et al. Uncovering burden disparity: a comparative analysis of the impact of moderate-to-severe psoriasis and hidradenitis suppurativa. J Am Acad Dermatol. 2017;77(6):1038-46.

20. Tzellos T, Yang H, Mu F, Calimlim B, Signorovitch J. Impact of hidradenitis suppurativa on work loss, indirect costs and income. Br J Dermatol. 2019;181: 147-54.

21. Prens LM, Huizinga J, Janse IC, Horváth B. Surgical outcomes and the impact of major surgery on quality of life, activity impairment and sexual health in hidradenitis suppurativa patients: a prospective single centre study. J Eur Acad Dermatol Venereol. 2019. https://doi.org/10.1111/jdv. 15706 .

22. Tzellos T, Song Y, Wang J, Yang H, Singh R, Calimlim B. Impact of adalimumab on trajectories of work productivity impairment, skin pain, and quality of life measures among patients with hidradenitis suppurativa. 28th EADV (October 9-13, 2019, Madrid), P0005.

23. Fauconier M, Reguiai Z, Barbe C, et al. Association between hidradenitis suppurativa and spondyloarthritis. Jt Bone Spine. 2018;85:593-7.

24. Rondags A, Arends S, Wink FR, Horváth B, Spoorenberg A. High prevalence of hidradenitis suppurativa symptoms in axial spondyloarthritis patients: a possible new extra-articular manifestation. J Eur Acad Dermatol Venereol. 2018;32: 1784-90.

25. Chen WT, Chi CC. Association of hidradenitis suppurativa with inflammatory bowel disease: a systematic review and meta-analysis. JAMA Dermatol. 2019. https://doi.org/10.1001/ jamadermatol.2019.0891.
26. Garg A, Hundal J, Strunk A. Overall and subgroup prevalence of crohn disease among patients with hidradenitis suppurativa: a population-based analysis in the United States. JAMA Dermatol. 2018;154: 814-8.

27. Ardon CB, Molenaar C, van Straalen KR, Scholtes VC, Prens EP, van der Zee HH. High prevalence of hidradenitis suppurativa in patients with perianal fistula. Int J Colorectal Dis. 2019;34:1337-9.

28. Von der Werth JM, Jemec GB. Morbidity in patients with hidradenitis suppurativa. $\mathrm{Br} \mathrm{J}$ Dermatol. 2001;144:809-13.

29. Matusiak L, Bieniek A, Szepietowski JC. Psychophysical aspects of hidradenitis suppurativa. Acta Dermatol Venereol. 2010;90:265-8.

30. Esmann S, Jemec GBE. Acta Derm Venereol. 2011;91:328-32.

31. Schneider-Burrus S, Jost A, Peters EMJ, WitteHaendel E, Sterry W, Sabat R. Association of hidradenitis suppurativa with body image. JAMA Dermatol. 2018;154:447-51.

32. Janse IC, Deckers IE, van der Maten AD, et al. Sexual health and quality of life are impaired in hidradenitis suppurativa: a multicentre cross-sectional study. Br J Dermatol. 2017;176:1042-7.

33. Alavi A, Farzanfar D, Rogalska T, Lowes MA, Chavoshi S. Quality of life and sexual health in patients with hidradenitis suppurativa. Int J Womens Dermatol. 2018;4:74-9.

34. Machado MO, Stergiopoulos V, Maes M, et al. Depression and anxiety in adults with hidradenitis suppurativa: a systematic review and meta-analysis. JAMA Dermatol. 2019. https://doi.org/10.1001/ jamadermatol.2019.0759.

35. Tzur Bitan D, Berzin D, Cohen AD. Hidradenitis suppurativa (HS) and schizophrenia: a nationwide cohort study. J Eur Acad Dermatol Venereol. 2019. https://doi.org/10.1111/jdv.15997.

36. Thorlacius L, Cohen AD, Gislason GH, Jemec GBE, Egeberg A. Increased suicide risk in patients with hidradenitis suppurativa. J Invest Dermatol. 2018;138:52-7.

37. Vinkel C, Thomsen SF. Autoinflammatory syndromes associated with hidradenitis suppurativa and/or acne. Int J Dermatol. 2017;56:811-8.

38. Reddy S, Orenstein LAV, Strunk A, Garg A. Incidence of long-term opioid use among opioid-naive patients with hidradenitis suppurativa in the United States. JAMA Dermatol. 2019. https://doi.org/ 10.1001/jamadermatol.2019.2610. 
39. Kjaersgaard Andersen R, Saunte SK, Jemec GBE, Saunte DM. Psoriasis as a comorbidity of hidradenitis suppurativa. Int $\mathrm{J}$ Dermatol. 2019. https://doi.org/10.1111/ijd.14651.

40. Horissian M, Maczuga S, Kirby JS, Nelson AM. Increased risk of alopecia areata for people with hidradenitis suppurativa in a cross-sectional study. J Am Acad Dermatol. 2019. https://doi.org/ 10.1016/j.jaad.2019.05.093.

41. Lee JH, Kwon HS, Jung HM, Kim GM, Bae JM. Prevalence and comorbidities associated with hidradenitis suppurativa in Korea: a nationwide population-based study. Semin Arthritis Rheum. 2019;48:611-7.
42. Kohorst JJ, Kimball AB, Davis MD. Systemic associations of hidradenitis suppurativa. J Am Acad Dermatol. 2015;73:27-35.

43. Zouboulis CC, Tzellos T, Kyrgidis A, et al. Development and validation of the International Hidradenitis Suppurativa Severity Score System (IHS4), a novel dynamic scoring system to assess HS severity. Br J Dermatol. 2017;177:1401-9.

44. Kimball AB, Tzellos T, Calimlim BM, Teixeira HD, Geng Z, Okun MM. Achieving Hidradenitis Suppurativa Response Score is associated with significant improvement in clinical and patient-reported outcomes: post hoc analysis of pooled data from PIONEER I and II. Acta Derm Venereol. 2018;98:932-7. 